\title{
Long-Lasting Effects of Escalating Doses of $d$-Amphetamine on Brain Monoamines, Amphetamine-Induced Stereotyped Behavior and Spontaneous Nocturnal Locomotion
}

\author{
TERRY E. ROBINSON AND DIANNE M. CAMP \\ Department of Psychology and Neuroscience Laboratory Building. The University of Michigan \\ II03 E. Huron St. Ann Arbor, MI 48104-1687
}

Received 1 August 1986

\begin{abstract}
ROBINSON. T. E. AND D. M. CAMP. Long-lasting effects of escalating doses of d-amphetamine on brain monoamines. amphetamine-induced stereotyped behavior and spontaneous nocturnal locomotion. PHARMACOL BIOCHEM BEHAV 26 (4) 821-827. 1987. - The repeated intermittent administration of relatively low doses of amphetamine (AMPH) produces an enduring hypersensitivity to the motor stimulant effects of AMPH (behavioral sensitization), and this is accompanied by enhanced mesotelencephalic dopamine (DA) utilization/release. In contrast, chronic treatment with very high doses of AMPH does not produce sensitization. but is neurotoxic, resulting in the depletion of brain DA (and often other monoamines). However, gradually escalating doses of AMPH provide protection against the neurotoxic effects of higher doses given later. Therefore, the purpose of the present experiment was to determine if a regimen of gradually escalating doses of AMPH, culminating in much higher doses than usually used to study sensitization, would produce neural and behavioral changes associated with AMPH neurotoxicity (DA depletion) or behavioral sensitization (increased DA utilization). Female rats were given 60 injections $(2 /$ day) of increasing (1 to $10 \mathrm{mg} / \mathrm{kg}$ ) doses of $d-\mathrm{AMPH}$, culminating in rats receiving $20 \mathrm{mg} / \mathrm{kg} /$ day for four consecutive days. This treatment did not deplete brain DA or serotonin, but did produce a long-lasting enhancement (at least 12 days) in striatal and nucleus accumbens DOPAC concentrations, and DOPAC/DA ratios. These neurochemical changes were accompanied by an enduring hypersensitivity to the stereotypy-producing effects of a subsequent AMPH 'challenge.' In contrast to this enhanced response to a challenge, AMPH-pretreated rats showed a marked reduction in spontaneous nocturnal motor activity. It is concluded that rats can be given escalating doses of AMPH, which mimic to some extent the AMPH 'runs' common in addicts and that this produces neural and behavioral changes consistent with the development of sensitization; not neurotoxicity.
\end{abstract}

Amphetamine Sensitization Locomotion Motor activity Neurotoxicity Dopamine Striatum Nucleus accumbens Frontal cortex Serotonin Chronic treatment Amphetamine psychosis

Dopamine metabolism

IN humans chronic amphetamine (AMPH) use frequently produces an AMPH psychosis with clinical features that are largely indistinguishable from paranoid schizophrenia [7,15]. In addition, former AMPH addicts remain hypersensitive to the psychotomimetic effects of AMPH even after years of abstinence $[37,41]$, suggesting that AMPH may produce persistent changes in neural regions that mediate the psychotomimetic effects of AMPH. These clinical observations have prompted considerable interest in the long-term effects of stimulant drugs on brain and behavior in nonhu- man animals, and in the development of animal models of AMPH psychosis.

One syndrome that has been proposed as an animal model of AMPH psychosis is called 'behavioral sensitization' $([17,42]$, for reviews see $[32,41])$. This refers to the progressive and long-lasting hypersensitivity to the motor stimulant effects of AMPH produced by the repeated intermittent administration of AMPH. A second syndrome associated with chronic AMPH treatment will be called 'AMPH neurotoxicity,' and this refers to the progressive changes in 
brain and behavior produced by continuously maintaining elevated blood levels of AMPH for many days at a time $[11,32]$. This is usually achieved by implantation of a subcutaneous 'pellet' that slowly releases AMPH [10], or by the frequent injection of very high doses of AMPH [23, 29, 43]. The rationale for frequently administering high doses of AMPH to animals is that chronic AMPH users often take large quantities of AMPH in 'runs' that can last for 3-6 days [19], and researchers want to mimic this pattern of drug use [11]. However, as pointed out by Schmidt et al. [39], chronic AMPH users build up to high doses gradually, as they develop tolerance to AMPH's autonomic effects. Therefore, the abrupt administration of high doses of AMPH to nonhuman animals does not take into account changes in the effects of AMPH that may be associated with gradually escalating doses $[38,39]$.

Most studies on the neurological consequences of chronic AMPH treatment have focused on mesotelencephalic dopamine (DA) systems, because AMPH causes DA release, and many of the behaviors evoked by AMPH are thought largely to be due to changes in DA activity. The repeated intermittent administration of relatively low doses of AMPH ( $\leqslant 5.0 \mathrm{mg} / \mathrm{kg}$ ), which produces behavioral sensitization, is associated with long-lasting increases in stimulated striatal DA release in vitro $[5,18,31,34]$, and in nucleus accumbens and medial frontal cortex DA utilization [4, 24, 33]; but no changes in DA concentrations [32]. In contrast, the continuous infusion of AMPH, or frequent injection of multiple high doses, depletes striatal DA (and often serotonin). presumably due to terminal degeneration $[10,23,28,29.43]$. Furthermore, there appears to be a reduction in the ability of a challenge injection of AMPH to enhance dopaminergic activity in animals pretreated with toxic doses of AMPH [38]. However, it appears that prior exposure to gradually increasing doses of AMPH provides considerable protection against the neurotoxic effects of high doses given later [39]. Therefore, the purpose of the present experiment was to determine if a regimen of gradually escalating doses of $d$-AMPH, culminating in much higher doses than usually used to study sensitization, would produce neural and behavioral changes usually associated with AMPH neurotoxicity or behavioral sensitization. In addition, spontaneous nocturnal motor activity was studied following the cessation of AMPH treatment to determine if normal activity rhythms were changed by prior exposure to AMPH.

\section{METHOD}

Thirty adult (200-300 g) female Sprague-Dawley derived rats (Holtzman Co., Madison, WI) were housed individually in wire-hanging cages in a temperature-controlled room, on a normal light:dark cycle (14:10 hr, with the lights going on at 08:00 hr). Food and water were freely available.

Each rat received two daily intraperitoneal injections of either $0.9 \%$ saline $(1 \mathrm{~mL} / \mathrm{kg} ; \mathrm{n}=13)$ or $d$-amphetamine sulfate (AMPH), with 8-10 hr separating the two daily injections. Immediately following the injection each rat was returned to its home cage. Injections were given on each consecutive weekday for 6 weeks, but not on weekends (i.e., there were two drug-free days after each 10 consecutive injections). AMPH:pretreated rats $(n=17)$ received increasing doses of AMPH for a total of 60 injections over 42 days $(30$ injection days), according to the following schedule: Injection days $1-2(1.0 \mathrm{mg} / \mathrm{kg}) ; 3-5(2.0 \mathrm{mg} / \mathrm{kg}) ; 6(3.0 \mathrm{mg} / \mathrm{kg}) ; 7-11(4.0$ $\mathrm{mg} / \mathrm{kg}) ; 12-15(5.0 \mathrm{mg} / \mathrm{kg}) ; 16(6.0 \mathrm{mg} / \mathrm{kg}) ; 17-20(7.0 \mathrm{mg} / \mathrm{kg})$;
$21(8.0 \mathrm{mg} / \mathrm{kg}) ; 22-26(9 \mathrm{mg} / \mathrm{kg})$; and $27-30(10 \mathrm{mg} / \mathrm{kg})$. Therefore, over the last 4 days of the treatment schedule AMPHpretreated rats received two daily injections of $10 \mathrm{mg} / \mathrm{kg}$. The animals were then used in either the neurochemical or behavioral study described below.

\section{Neurochemical Study}

Twelve days after the last injection of AMPH or saline, during which time the animals were left undisturbed, ten of the AMPH-pretreated and six of the saline-pretreated rats were decapitated. After each brain was rapidly removed $(<30 \mathrm{sec})$ it was immediately put in ice-cold saline for $30-45$ $\mathrm{sec}$ and then placed in a chilled cutting block. Brain slices were obtained as described by Heffner et $a l$. [12], but with the following modifications. (1) Only the anteromedial portion of the frontal cortex anterior to the genu of the corpus callosum was removed (the dorsolateral cortex was discarded). (2) The corpus of the striatum was removed with a $3.0 \mathrm{~mm}$ diameter micropunch. (3) The nucleus accumbens was removed with a $2.0 \mathrm{~mm}$ diameter micropunch. Tissue from the left and right hemispheres was pooled.

As the dissection of each region was completed, the tissue pieces were weighed, placed in tubes containing $0.05 \mathrm{~N}$ perchloric acid and dihydroxybenzylamine (DHBA; internal standard), and then homogenized. Samples were centrifuged at $5,000 \times \mathrm{g}$ for $45 \mathrm{~min}$ at $2-4^{\circ} \mathrm{C}$, the supernatant filtered through Arco LC3A $0.45 \mu \mathrm{m}$ pore filters (Gelman Sciences, Ann Arbor) and then stored frozen at $-20^{\circ} \mathrm{C}$ for no more than 2 weeks before being assayed. Samples for a six point standard curve were prepared in the perchloric acid/DHBA solution at the same time. Tissue concentrations of dopamine (DA), dihydroxyphenylacetic acid (DOPAC), 5hydroxytryptamine (5-HT) and 5-hydroxyindoleacetic acid (5-HIAA) were subsequently determined by high performance liquid chromatography with electrochemical detection, using procedures similar to those described previously [3].

\section{Behavioral Studies}

The remaining animals ( 7 saline- and 7 AMPH-pretreated) were left undisturbed in their home cages for 2 days following the last pretreatment injection, and were then transferred to wire-hanging cages adapted for measuring motor activity. These cages were similar to the home cages, but twice as long ('double' cages: $41 \times 24 \times 18 \mathrm{~cm}$ ), and were equipped with two pairs of infrared photocells situated along the long axis of the cage. The photocell pairs were mounted $5.0 \mathrm{~cm}$ above the cage floor and $25.2 \mathrm{~cm}$ apart from each other: thus dividing the cage into a central area $(25.2 \mathrm{~cm}$ long) in which there was no photocell beam, and two $7.9 \mathrm{~cm}$ long areas to the extreme left and right ends of the cage, in which the presence of a rat would result in disruption of a photocell beam. Disruption of a photocell beam was detected by a microcomputer, which registered the total number of beam 'breaks' over any specified interval. Disrupting one beam registered a single count, but another count could not be registered by that beam until the second photocell beam at the other end of the cage was disrupted. Therefore, activity counts in this apparatus reflect locomotion from one end of the cage to the other. and not the repetitive disruption of one beam.

The animais remained in these activity monitors for 24 hr/day for the next 10 days. The animals were left to habituate for the first 5 days, but for the next 5 days activity was monitored over $30 \mathrm{~min}$ intervals for $20 \mathrm{hr} /$ day. The light: 
TABLE 1

MEAN ( \pm S.E.M.) TISSUE CONCENTRATIONS (ng/mg) OF DOPAMINE, DIHYDROXYPHENYLACETIC ACID (DOPAC). 5-HYDROXYTRYPTAMINE (5-HT), 5-HYDROXYINDOLEACETIC ACID (5-HIAA). AND THE MEAN DOPACIDA AND 5-HIAA/5-HT RATIOS IN SALINE AND AMPHETAMINE (AMP)-PRETREATED RATS

\begin{tabular}{|c|c|c|c|c|c|c|}
\hline & Dopamine & DOPAC & DOPAC/DA & $5-\mathrm{HT}$ & 5-HIAA & 5-HIAA/5-HT \\
\hline \multicolumn{7}{|l|}{ Striatum } \\
\hline Saline (5) & $21.37 \pm 0.81$ & $2.23 \pm 0.18$ & $0.104 \pm 0.01$ & $0.824 \pm 0.10$ & $0.959 \pm 0.08$ & $1.19 \pm 0.06$ \\
\hline AMP (10) & $21.47 \pm 0.81$ & $3.03 \pm 0.21^{*}$ & $0.140 \pm 0.01 \dagger$ & $0.746 \pm 0.09$ & $0.923 \pm 0.06$ & $1.36 \pm 0.15$ \\
\hline \multicolumn{7}{|c|}{ Nucleus Accumbens } \\
\hline Saline (6) & $14.03 \pm 0.12$ & $2.92 \pm 0.32$ & $0.206 \pm 0.01$ & $0.828 \pm 0.13$ & $0.910 \pm 0.09$ & $1.19 \pm 0.17$ \\
\hline AMP (10) & $15.77 \pm 0.08$ & $3.72 \pm 0.21^{*}$ & $0.236 \pm 0.01^{*}$ & $0.983 \pm 0.13$ & $1.028 \pm 0.11$ & $1.09 \pm 0.10$ \\
\hline \multicolumn{7}{|c|}{ Medial Frontal Cortex } \\
\hline Saline $(6)$ & $0.178 \pm 0.02$ & $0.168 \pm 0.03$ & $1.00 \pm 0.19$ & $1.120=0.05$ & $0.643 \pm 0.05$ & $0.58 \pm 0.05$ \\
\hline AMP (10) & $0.228 \pm 0.01$ & $0.176=0.02$ & $0.82=0.14$ & $0.979=0.09$ & $0.650 \pm 0.03$ & $0.75 \pm 0.10$ \\
\hline
\end{tabular}

*Differs from the saline control group, $p<0.05 ; \dagger p<0.01$. Number in parentheses $=$ number of animals.

dark cycle was the same as in the home colony room. Twelve days after the last pretreatment with saline or AMPH all animals received a challenge injection (IP) of $2.0 \mathrm{mg} / \mathrm{kg}$ $d$-amphetamine sulfate, and AMPH-induced stereotyped behavior and locomotion (photocell disruptions over $5 \mathrm{~min}$ intervals) were quantified over the next $2 \mathrm{hr}$.

Stereotyped behavior was rated by an observer (D.M.C.), who was unaware of the pretreatment conditions, every 10 min following the injection of AMPH. Overall stereotypy was assessed by use of the 9-point scale described by Ellinwood and Balster [9], in which a score of $1=$ asleep, $2=$ inactive, $3=$ normal in place activity, $4=$ normal, alert, active, $5=$ hyperactive, $6=$ slow patterned stereotypy, $7=$ fast patterned stereotypy, $8=$ restricted (focussed) stereotypy, and $9=$ dyskinetic (see Ellinwood and Balster [9] for a more complete description of the rating scale). In addition, subcomponents of stereotyped activity, including repetitive sniffing behavior, repetitive movements of the head and limbs and repetitive oral behaviors (licking and biting) were rated according to their intensity $(1=$ mild, $2=$ moderate, $3=$ intense) and duration ( $1=$ discontinuous, $2=$ continuous $)$, as described by Rebec and Segal [26]. For each subcomponent of stereotypy the intensity and duration scores were multiplied together to yield a single rating at each time interval.

\section{RESULTS}

\section{Neurochemistry}

Table 1 presents the mean concentrations of DA, DOPAC, 5-HT and 5-HIAA in the striatum, nucleus accumbens and medial frontal cortex of AMPH-pretreated and control animals. The striatal sample from one control animal was lost, and that is why there are only 5 animals in that group. The DOPAC/DA and 5-HIAA/5-HT ratios, which provide an index of DA and 5-HT utilization, respectively [27, 36, 44], are also shown.

AMPH-pretreatment had no affect on DA, 5-HT or 5-HIAA concentrations, or 5-HIAA/5-HT ratios, in any of the structures sampled (2-tailed $t$-tests). However, AMPHpretreatment did produce a significant increase in DOPAC concentrations in the striatum, $t(13)=2.5, p=0.026$, and nucleus accumbens, $t(14)=2.2, p=0.043$, but not in the medial frontal cortex. Similarly, AMPH-pretreatment significantly

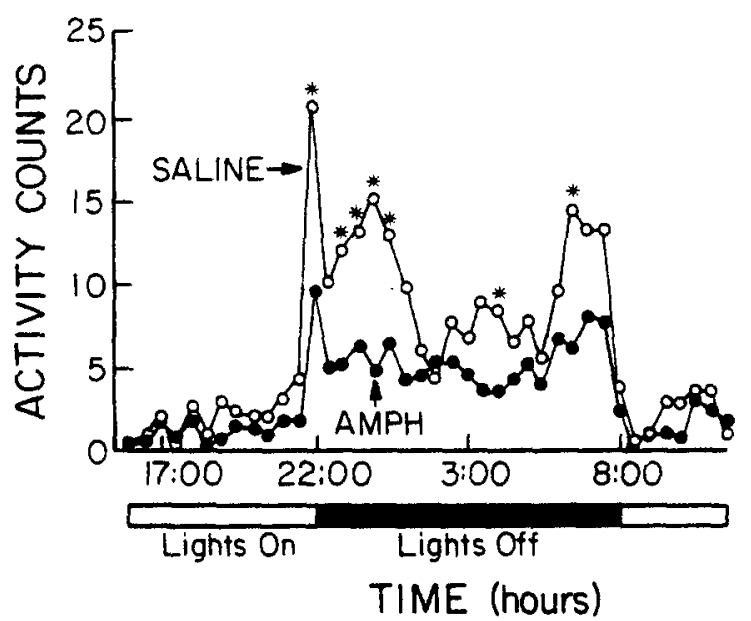

FIG. 1. Mean spontaneous activity counts averaged over $30 \mathrm{~min}$ intervals for 20 consecutive hours (16:00 to 12:00 hr) in amphetamine (AMPH)- and saline-pretreated rats ( $n=7 /$ group). In this apparatus activity counts reflect locomotion from one end of the cage to the other (see the Method section). AMPH-pretreated rats were significantly less active than saline controls, as indicated by a two-way ANOVA with repeated measures over all 20 intervals (Main Effect $\mathrm{F}=28.3, p<0.001$; Interaction $\mathrm{F}=4.5, p<0.001$ ). Analyses comparing the two groups just during the dark part of the light:dark cycle (lights off). or just during the $6 \mathrm{hr}$ prior to lights off were also statistically significant (ANOVAs, $p<0.05$ ). Asterisks indicate individual comparisons significant at the $p<0.01$ level $(t$-tests)

increased DOPAC/DA ratios in the striatum, $t(13)=3.6$, $p=0.003$, and nucleus accumbens, $t(14)=2.4, p=0.03$, but not medial frontal cortex (Table 1).

\section{Behavior}

Spontaneous activity. Figure 1 illustrates the averaged spontaneous activity of saline and AMPH-pretreated rats over a $20 \mathrm{hr}$ period. Data from the remaining $4 \mathrm{hr} /$ day were not included to minimize disturbances associated with feeding, watering, cage-cleaning and data collection (printing) that occurred during this period. The data shown in Fig. 1 were averaged over the 5 consecutive days of testing to re- 
OVERALL STEREOTYPY
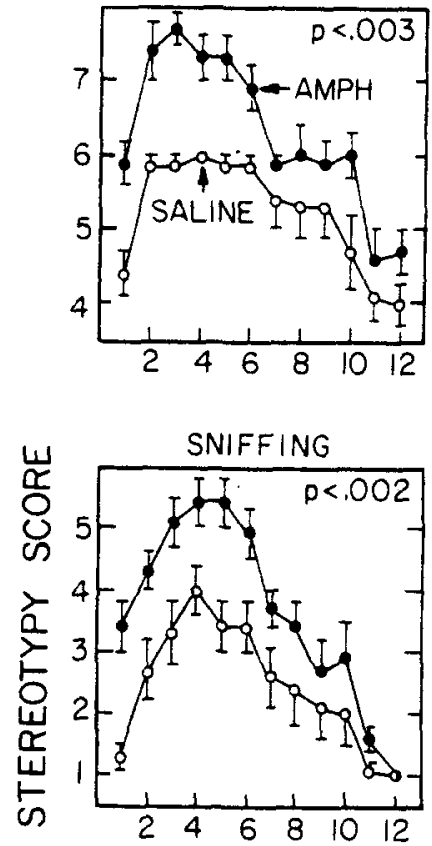

HEAD \& LIMB MOVEMENTS

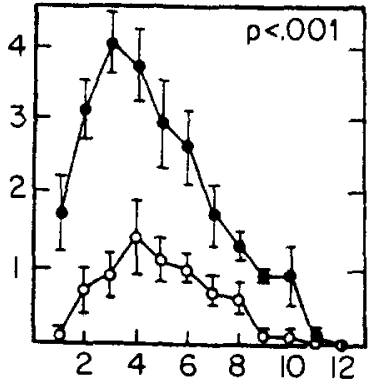

TEN MINUTE INTERVALS

FIG. 2. Mean ( \pm S.E.M.) stereotypy rating scores for AMPH-and saline-pretreated rats $(n=7 /$ group). Twelve days after the last treatment with saline or AMPH each rat received a challenge injection of $2.0 \mathrm{mg} / \mathrm{kg} d$-AMPH, and then were rated once every $10 \mathrm{~min}$ for the next $2 \mathrm{hr}$. Mann-Whitney U-tests were conducted on the cumulative rating scores, and AMPH-pretreated rats showed significantly greater overall stereotypy (top; $U=4, p<0.003$ ), repetitive sniffing (middle; $\mathrm{U}=3, p<0.002$ ), and repetitive head and limb movements (bottom: $\mathrm{U}=1.5, p<0.001$ ) than did saline-pretreated controls.

duce intra-subject variability associated with the estrous cycle. Each rat would have had one and only one complete estrous cycle during this time. Exactly the same pattern of results was present when data for each day were analyzed separately.

Both groups showed the same pattern of activity over the light:dark cycle: i.e., relatively low levels of activity during the day; a large increase immediately following lights off; a reduction in activity during the middle of the dark period; a second rise in activity towards the end of the dark period: and then a marked decline with lights on (Fig. 1). During the light period there were only small differences in activity between the $\mathbf{2}$ groups, but there were large group differences in nocturnal activity. At night AMPH-pretreated rats were markedly less active than saline-pretreated controls, and this was especially evident during the period immediately following lights off, and just prior to lights on (Fig. 1). During the middle of the dark period (around interval 20) both groups showed similar relatively low levels of activity, but were still more active than during the day.

AMPH-induced behavior. As described previously (e.g., [42]), a $2.0 \mathrm{mg} / \mathrm{kg}$ challenge injection of AMPH produced a typical triphasic pattern of behavioral change consisting of: (1) an initial enhancement in locomotor activity; (2) followed by a period of stereotyped behavior during which locomotor activity was much diminished; and (3) a post-stereotypy period of heightened locomotion. However, the response of AMPH-pretreated rats was significantly different from that of saline-pretreated controls, and the difference was typical of that associated with sensitization to AMPH. Figure 2 illustrates the stereotypy ratings obtained over $2 \mathrm{hr}$ following the AMPH challenge, and it is obvious that AMPH-pretreated rats showed a more rapid onset of stereotyped behavior and more intense stereotyped behavior than did saline-pretreated controls. This dose of AMPH did not produce oral stereotypies in any of the saline-pretreated rats, but $3 / 7$ of the AMPH-pretreated rats did show periods of licking and gnawing. Although the data are not shown. analysis of activity counts (photocell beam disruptions) resulted in the characteristic differences between AMPH- and saline-pretreated animals $(F=2.5, p<0.001)$ described extensively by Segal and colleagues $[41,42]$, i.e., a more rapid onset of stereotypy (cessation of locomotion) in AMPH-pretreated animals.

Lastly, the AMPH treatment regimen did not significantly disrupt growth rate in these animals, and if anything there was a tendency for AMPH-pretreated rats to gain more weight than saline controls. On the first treatment day the saline group weighed an average ( \pm SEM) of $193 \pm 4.5 \mathrm{~g}$ and the AMPH group $194 \pm 2.4 \mathrm{~g}$; on the last treatment day they weighed $267 \pm 10.1$ and $276 \pm 6.4 \mathrm{~g}$, respectively: and on the challenge day $275 \pm 10.8$ and $295 \pm 1.9 \mathrm{~g}$, respectively.

\section{DISCUSSION}

In the present study female rats received 60 injections of increasing doses of $A M P H$, culminating with rats receiving $20 \mathrm{mg} / \mathrm{kg} /$ day for 4 consecutive days. This treatment regimen was designed to mimic, to some extent, the pattern of drug use seen in AMPH addicts [19]. In this regard it is worth noting that a $65 \mathrm{~kg}$ human taking $20 \mathrm{mg} / \mathrm{kg} /$ day would use 1.3 grams/day. The purpose of this study was to determine if this regimen of gradually escalating doses of AMPH would produce neurochemical and behavioral changes normally associated with the phenomenon of behavioral sensitization. or with the phenomenon of AMPH neurotoxicity ([32] for review). Both the neurochemical and behavioral changes observed are clearly consistent with the induction of sensitization, and not with AMPH neurotoxicity.

\section{Nerorochemical Changes}

It is well established that the continuous infusion or repeated administration of very high doses of $A M P H$ is toxic to monoaminergic neurons, and that this is typically manifest by a persistent depletion of forebrain DA and/or 5-HT. presumably due to terminal degeneration $[10,23,25,28,29$. $38.39,43$ ]. Doses comparable to those used here have been reported to be sufficient to produce a long-lasting depletion of striatal DA. for example, twice daily SC injections of 12.5 $\mathrm{mg} / \mathrm{kg} d$-AMPH for 4 days [40]. However, the present treat- 
ment regimen was not toxic to monoaminergic neurons. as indicated by normal tissue concentrations of DA and 5-HT in the striatum, nucleus accumbens and medial frontal cortex. There are probably a number of reasons for this. (1) Monoamine neurons are particularly sensitive to damage when elevated blood levels of AMPH are continuously maintained. and less sensitive to intermittent injections. as used here [32]. (2) In the present study animals were given gradually escalating doses of AMPH, and it has been shown this provides considerable protection against the toxic effects of subsequent high doses [39]. (3) In many studies describing AMPH neurotoxicity, especially in which 5-HT systems were damaged, meth-AMPH was used. In rats meth-AMPH is probably more toxic than d-AMPH [25.32]. Nevertheless, the present study clearly shows that rats can be given repeated high doses of $d$-AMPH without producing neurotoxic effects.

Although this AMPH treatment regimen did not deplete forebrain DA, it did enhance striatal and nucleus accumbens DA metabolism, as indicated by elevated DOPAC concentrations. The increase in DOPAC concentrations, and DOPAC/DA ratios, suggests that AMPH pretreatment produced a long-lasting (at least 12 days) increase in striatal and nucleus accumbens DA utilization. The ratio of DOPAC/DA does not always provide a good index of DA utilization, because after the administration of many psychoactive drugs DA metabolism can be dissociated from DA utilization/release $[6,47]$. However, in nondrugged animals. such as those studied here, there is considerable evidence that DOPAC/DA ratios provide a reasonably good index of DA utilization $[21,27,36]$.

The suggestion that this AMPH treatment regimen enhanced DA utilization is consistent with some studies on the neurochemical correlates of behavioral sensitization. For example, it has been reported that sensitization is accompanied by an enduring enhancement in AMPH [18, 31, 34], potassium and electrical stimulation-induced striatal DA release [5], and in striatal DA metabolism [4.46]. However. there have been numerous reports that sensitization does not alter steady-state (resting) levels of DA metabolism [14. 20, $22,24]$ but only the response to a subsequent challenge [32]. Of course, differences between these latter studies and the present one could be due to the relatively extreme AMPH treatment regimen used here. However, another possibility is that female rats were used in the present study, and in most previous studies with negative results male rats were used. Consistent with this idea, we have found that the behavioral sensitization produced by a much less extreme AMPH treatment regimen than used here is accompanied by enhanced striatal DA metabolism in female. but not male rats [4]. This sex difference in the persistent neurochemical effects of repeated AMPH treatment may be related to sex differences in the development of behavioral sensitization. Females show much more robust behavioral sensitization than males $[4,30,34]$. This is not to say that AMPH treatment produces qualitatively different effects in males and females, because both sensitized males and females are hyperresponsive to an AMPH challenge [5. 18, 31, 34]. Furthermore, a subgroup of male rats showing the most robust behavioral sensitization do have increased striatal DA metabolism (unpublished observations). Therefore, it is most likely that the sex difference is quantitative, and that relatively small changes during the resting state are more consistently apparent in highly sensitized females [32].

There was no effect of AMPH pretreatment on medial frontal cortex DA metabolism in the present study. This deserves mention because we previously reported that sensitization to AMPH is accompanied by an increase in medial frontal cortex DA utilization [33]. When we have estimated the 'resting' rate of DA utilization by measuring the decline in DA after an injection of alpha-methyl-p-tyrosine we have consistently (two independent experiments) found that sensitized rats show enhanced DA utilization in the medial frontal cortex. but not in the striatum [33]. In contrast, when we have measured 'resting' DA metabolite concentrations and metabolite/transmitter ratios we have consistently found that sensitized female rats show enhanced DA metabolism in the striatum. but not medial frontal cortex $([4]$; and the present study). It is not known what accounts for this discrepancy. It may be related to the large regional differences in the basal rate of DA utilization [2]. and/or regional differences in the sensitivity to stress (e.g.. [35.45] and below).

\section{Behavion}

The behavioral response to a challenge injection of AMPH observed here was typical of that frequently described in animals sensitized to AMPH [32]. AMPHpretreated rats showed a more rapid onset of stereotyped behavior, and more intense stereotyped movements than did saline controls. These behavioral observations further support the contention that the gradually escalating doses of AMPH used here produced sensitization.

The marked nocturnal hypoactivity shown by AMPHpretreated animals was unexpected. To our knowledge, this is the first report of abnormalities in the spontaneous behavior of AMPH-sensitized animals, which persists for more than a week after the cessation of drug treatment. Typically, behavioral abnormalities in AMPH-sensitized animals have been observed only following a challenge, either with AMPH or stress [32]. The decrease in nocturnal activity shown by AMPH-pretreated rats is especially striking when contrasted with the exaggerated behavioral response produced by an AMPH challenge. It is not known how long this nocturnal hypoactivity persists, or whether it is produced by less extreme AMPH treatment regimens. However, if the neural changes responsible for the decrease in spontaneous activity are related to those responsible for behavioral sensitization. the effect may persist for very long periods of time.

Although the hypoactivity seen in AMPH-pretreated animals was by far most pronounced during the dark part of the light:dark cycle, there was also a tendency for them to be relatively inactive during the lights-on period as well. This effect was very small but statistically significant when all the observation periods before lights off (i.e., the first $6 \mathrm{hr}$ in Fig. 1). were included in an analysis of variance (group $\mathrm{F}=7.0, p<0.03$ ). At the very least, AMPH-pretreated animals were not more active than controls during the day. This observation prompts speculation regarding the significance of the enhancement in resting DA metabolism found in the striatum and nucleus accumbens of AMPH sensitized rats.

Present knowledge concerning the role of mesostriatal and mesolimbic DA neurons in controlling motor activity suggests that an enhancement in dopaminergic activity should be accompanied by an increase in motor activity, not the hypoactivity reported here [13]. Of course, this apparent paradox may simply reflect a lack of correlation between the measures of DA metabolism used here, and DA utilization $[6,47]$. However, if the enhancement in DOPAC concentra- 
tions and DOPAC/DA ratios in fact do reflect increased DA utilization, why is this accompanied by apparent hypoactivity? The answer to this question is unknown, but one possibility is that the neurochemical measures reported here do not in fact reflect the resting state, but a rapid response to stress.

In the present study 2-3 min could pass after a rat was removed from its home cage before it was decapitated. This amount of time may be enough for the emergence of stressrelated changes in DA metabolism (utilization?). This idea is consistent with reports that intense environmental stimuli (stressors) can produce very rapid (a few min) increases in dopaminergic activity $[8,16,21,35]$. Furthermore, AMPHpretreated rats are hypersensitive to stress, as indicated by behavioral [1] and neurochemical studies [35]. Therefore, sensitized animals may show an even more rapid and intense response to removal from the home cage, and the associated handling, than normally expected. If this is the case, the enhanced striatal and nucleus accumbens DA metabolism reported here could reflect the response to a 'stress challenge,' consistent with the behavioral hypersensitivity to the AMPH challenge, rather than reflecting the resting state. Of course, it follows from this argument that if sensitized animals are left undisturbed, some DA systems may actually be hypoactive, which would coincide with a reduction in spontaneous activity. This reduction in motor activity could also be related to the small decrease in DA receptor binding frequently reported in AMPH pretreated rats [32]. It is interesting to speculate that such a reduction in dopaminergic activity could be responsible for the dysphoric 'crash' that follows AMPH (or cocaine) use, leading to the very strong desire for more. Of course, reexposure to AMPH would lead to an exaggerated dopaminergic response (sensitization), perhaps related to the paranoid psychotic reaction that so frequently develops with repeated stimulant drug use $[7,15$, $41]$.

In summary, the present study shows that rats can be given a regimen of escalating doses of $d$-AMPH, culminating in the repeated administration of very high doses, without producing neurotoxic effects. Animals given this treatment regimen showed enduring behavioral and neurochemical changes associated with the development of sensitization, including enhanced stereotyped behavior following an AMPH challenge, and enhanced striatal and nucleus accumbens DA metabolism [32]. Finally, AMPH-pretreated animals also showed a marked reduction in spontaneous nocturnal motor activity (locomotion), which persisted for at least 12 days following the last exposure to AMPH. It is suggested that the persistent behavioral and neurochemical changes produced by repeated AMPH treatment found here may be related to some of the persistent behavioral and cognitive effects of AMPH reported in people who chronically abuse stimulant drugs.

\section{ACKNOWLEDGEMENTS}

This research was supported by grants from the NIH (No. MH37277), The Scottish Rite Schizophrenia Research Program and RCDA No. NS00844 to T. E. Robinson. We thank T. Urban and C. J. Moore for their assistance in conducting the experiment. S. Waltz for typing the manuscript and Dr. J. B. Becker for advice concerning assay procedures.

\section{REFERENCES}

1. Anteiman, S. M., A. J. Eichler, C. A. Black and K. Kocan. Interchangeability of stress and amphetamine in sensitization. Science 207: 329-331, 1980.

2. Bannon, M. J., E. B. Bunney and R. H. Roth. Mesocortical dopamine neurons: rapid transmitter turnover compared to other brain catecholamine systems. Brain Res 218: 376-382. 1981.

3. Becker, J. B., E. Castañeda. T. E. Robinson and M. E. Beer. A simple in vitro technique to measure the release of endogenous dopamine and dihydroxyphenylacetic acid from striatal tissue using high performance liquid chromatography with electrical detection. J Neurosci Methods 11: 19-28, 1984.

4. Camp, D. M. and T. E. Robinson. Sex differences in an animal model of amphetamine psychosis. Soc Nenrosci Abstr 11: 550, 1985.

5. Castañeda, E.. J. B. Becker and T. E. Robinson. Depolarization-induced striatal dopamine release in rats sensitized to amphetamine. Soc Veurosci Abstr 12: 1141, 1986.

6. Commissiong. J. W. Monoamine metabolites: their relationship and lack of relationship to monoaminergic neuronal activity. Biochem Pharmacol 34: 1127-1131. 1985.

7. Connell, P. H. Amphetamine Psychosis. London: Chapman and Hill, 1958.

8. Curzon. G., P. H. Hutson and P. J. Knott. Voltammetry in vivo: effect of stressful manipulations and drugs in the caudate nucleus of the rat. Br J Pharmacol 66: 127P-128P, 1979.

9. Ellinwood, E. H. and R. L. Balster. Rating the behavioral effects of amphetamine. Eur J Pharmacol 28: 35-41. 1974.

10. Ellison. G., M. S. Eison, H. S. Huberman and F. Daniel. Longterm changes in dopaminergic innervation of caudate nucleus after continuous amphetamine administration. Scicnc' 201: 276-278, 1978.
11. Ellison, G. D. and M. S. Eison. Continuous amphetamine intoxication: an animal model of the acute psychotic episode. Psychol hed 13: 751-762. 1983.

12. Heffner, T. G.. J. A. Hartman and L. S. Seiden. A rapid method for the regional dissection of the rat brain. Pharmacos Binchem Bcha' 13: 453-456, 1980.

13. Iversen. S. D. Brain dopamine systems and behavior. In: Handbook of Psychopharmacology. Vol 8 . edited by $L$. L. Iversen. S. D. Iversen and S. H. Snyder. New York: Plenum. 1977. pp. 333-384.

14. Jackson. D. M., R. C. Bailey, M. J. Christie, E. A. Crisp and J. $H$. Skerritt. Long-term $d$-amphetamine in rats: lack of a change in post-synaptic dopamine receptor sensitivity. Psychopharmacology (Borlin) 73: 276-280. 1981.

15. Janowsky, D. S. and C. Risch. Amphetamine psychosis and psychotic symptoms. Psychopharmacology (Berlin) 65: 73-77. 1979.

16. Keller, R. W.. E. M. Stricker and M. J. Zigmond. Environmental stimuli but not homeostatic challenges produce apparent increases in dopaminergic activity in the striatum: an analysis by in viv' voltammetry. Brain Re's 279: 159-170. 1983.

17. Klawans. H. L. and D. I. Margolin. Amphetamine-induced dopaminergic hypersensitivity in guinea pigs. Arch Gen Psyihiutry 32: 725-732, 1975

18. Kolta. M. G.. P. Shreve, V. De Souza and N. J. Uretsky. Time course of the development of the enhanced behavioral and biochemical responses to amphetamine after pretreatment with amphetamine. Veuropharmacology 24: 823-829. 1985.

19. Kramer, J. C.. V. S. Fischman and D. C. Littlefield. Amphetamine abuse. JA.MA 201: 305-309. 1967. 
20. Kuczenski, R. and N. J. Leith. Chronic amphetamine: Is dopamine a link in or mediator of the development of tolerance and reverse tolerance? Pharmacol Biochem Bchav 15: 405-413. 1981 .

21. Lavielle. S., J.-P. Tassin. A.-M. Thierry, G. Blanc. D. Herve. C. Barthelemy and J. Glowinski. Blockage by benzodiazepines of the selective high increase in dopamine turnover induced by stress in mesocortical dopaminergic neurons of the rat. Brain Res 168: 585-594, 1978.

22. Mittleman. G.. E. Castañeda. T. E. Robinson and E. S. Valenstein. The propensity for non-regulatory ingestive behavior is related to differences in dopamine systems: behavioral and biochemical evidence. Behav Neurosci 100: 213-220, 1986.

23. Morgan, M. E. and J. W. Gibb. Short-term and long-term effects of methamphetamine on biogenic amine metabolism in extra-striatal dopaminergic nuclei. Neuropharmacology 19: 989-995, 1980.

24. Nishikawa. T.. N. Mataga, M. Takashima and M. Toru. Behavioral sensitization and relative hyperresponsiveness of striatal and limbic dopaminergic neurons after repeated methamphetamine treatment. Eur J Pharmacol 88: 195-203, 1983.

25. Peat. M. A.. P. F. Warren. C. Bakhit and J. W. Gibb. The acute effects of methamphetamine, amphetamine and pchloroamphetamine on the cortical serotonergic system of the rat brain: evidence for differences in the effects of methamphetamine and amphetamine. Eur J Pharmacol 116: 11-16. 1985.

26. Rebec. G. V. and D. S. Segal. Apparent tolerance to some aspects of amphetamine stereotypy with long-term treatment. Pharmacol Biochem Behav 13: 793-797, 1980.

27. Reinhard, J. F. Estimation of neurotransmitter metabolism by liquid chromatography and electrochemical detection: a possible means for assessing in vivo correlates of altered receptor sensitivity. In: Neuroreceptors in Health and Disease, edited by J. Maswaha and W. J. Anderson. Basel: Karger, 1984, pp. 27-52.

28. Ricaurte. G. A.. L. S. Seiden and C. R. Schuster. Further evidence that amphetamines produce long-lasting dopamine neurochemical deficits by destroying dopamine nerve fibers. Brain Re's 303: 359-364, 1984.

29. Ridley. R. M.. H. F. Baker, F. Owen, A. J. Cross and T. J. Crow. Behavioural and biochemical effects of chronic amphetamine treatment in the vervet monkey. Psychopharmacology: (Berlin) 78: 245-251, 1982.

30. Robinson, T. E. Behavioral sensitization: characterization of enduring changes in rotational behavior produced by intermittent injections of amphetamine in male and female rats. Psychopharmacology (Berlin) 84: 466-475, 1984.

31. Robinson. T. E. and J. B. Becker. Behavioral sensitization is accompanied by an enhancement in amphetamine-stimulated dopamine release from striatal tissue in vitro. Eur $J$ Pharmacol 85: 253-254. 1982.

32. Robinson. T. E. and J. B. Becker. Enduring changes in brain and behavior produced by chronic amphetamine administration: a review and evaluation of animal models of amphetamine psychosis. Brain Re's Re' 11: 157-198, 1986.
33. Robinson, T. E., J. B. Becker, C. J. Moore. E. Castañeda and G. Mittleman. Enduring enhancement in frontal cortex dopamine utilization in an animal model of amphetamine psychosis. Brain Re's 343: 374-377, 1985.

34. Robinson. T. E.. J. B. Becker and S. K. Presty. Long-term facilitation of amphetamine-induced rotational behavior and striatal dopamine release produced by a single exposure to amphetamine: sex differences. Brain Res 253: 231-241. 1982.

35. Robinson. T. E., J. B. Becker, E. A. Young, H. Akil and E. Castañeda. The effects of footshock stress on regional brain dopamine metabolism and pituitary beta-endorphin release in rats previously sensitized to amphetamine. Neuropharmacology, in press, 1987.

36. Roth. R. H., C. L. Murrin and J. R. Walters. Central dopaminergic neurons: effects of alterations in impulse flow on the accumulation of dihydroxyphenylacetic acid. Eur J Pharmacol 36: 163-171, 1976.

37. Sato, M., C.-C. Chen. K. Akiyama and S. Otsuki. Acute exacerbation of paranoid psychotic state after long-term abstinence in patients with previous methamphetamine psychosis. Biol Psychiatry 18: 429-440, 1983.

38. Schmidt, C. J.. D. R. Gehlert, M. A. Peat, P. K. Sonsalla. G. R. Hanson. J. K. Wamsley and J. W. Gibb. Studies on the mechanism of tolerance to methamphetamine. Brain Res 343: 305313. 1985.

39. Schmidt. C. J., P. K. Sonsalla, G. R. Hanson, M. A. Peat and J. W. Gibb. Methamphetamine-induced depression of monoamine synthesis in the rat: development of tolerance. I Neurochem 44: $852-855,1985$

40. Schuster, C. R. and C. E. Johanson. Efficacy, dependence potential and neurotoxicity of anorectic drugs. In: Behavioral Pharmacology: The Current Status, edited by L. S. Seiden and R. C. Balster. New York: Alan R. Liss, 1985, pp. 263-279.

41. Segal, D. S., M. A. Geyer and M. A. Schuckit. Stimulantinduced psychosis: an evaluation of animal models. In: Essays in Neurochemistry and Neuropharmacology, Vol 5, edited by M. B. H. Youdim. W. Lovenberg, D. F. Sharman and J. R. Lagnado. London: John Wiley \& Sons, 1981, pp. 95-129.

42. Segal, D. S. and A. J. Mandell. Long-term administration of amphetamine: Progressive augmentation of motor activity and stereotypy. Pharmacol Biochem Behav 2: 249-255, 1974.

43. Seiden. L. S., M. W. Fischman and C. R. Schuster. Long-term methamphetamine induced changes in brain catecholamines in tolerant rhesus monkeys. Drug Alcohol Depend 1: 215-219, $75 / 76$.

44. Shannon, N. J., J. W. Gunnett and K. E. Moore. A comparison of biochemical indices of 5-hydroxytryptaminergic neuronal activity following electrical stimulation of the dorsal raphe nucleus. I Neurochem 47: 958-965, 1986.

45. Thierry, A. M., J. P. Tassin, G. Blanc and J. Glowinski. Selective activation of the mesocortical DA system by stress. Nature 263: 242-244, 1976

46. Watanabe, H. Subchronic methamphetamine treatment selectively attenuates apomorphine-induced decrease in 3.4-dihydroxyphenylacetic acid level in mesolimbic dopaminergic regions. Life Sci 37: 2319-2325, 1985.

47. Westerink, B. H. Sequence and significance of dopamine metabolism in the rat brain. Neurochem Int 7: 221-227, 1985. 